\title{
Test of diurnal and semidiurnal tidal analysis of temperatures from SABER-like sampling of a realistic global model, CMAM-DAS
}

\author{
C. E. Meek ${ }^{1}$, A. H. Manson ${ }^{1}$, and J. R. Drummond ${ }^{2}$ \\ ${ }^{1}$ Institute of Space and Atmospheric Studies, University of Saskatchewan, Saskatoon, S7N 5E2, Canada \\ ${ }^{2}$ Department of Physics and Atmospheric Science, Dalhousie University, Halifax, B3H 3J5, Canada
}

Received: 18 February 2011 - Revised: 25 April 2011 - Accepted: 28 April 2011 - Published: 3 May 2011

\begin{abstract}
Because the TIMED satellite orbit precesses slowly in local time, 3 degrees $(12 \mathrm{~min})$ per day, thus apparently covering all local times at low and mid latitudes in 2 months when ascending and descending nodes are included, there is a potential for determining migrating and non-migrating tides from temperatures. Many papers have been written on the subject, but none have taken a realistic model atmosphere with high time resolution and tested tidal analysis. The present paper contains results from a tidal analysis of a SABER-sampled global model, the Canadian Middle Atmosphere (data assimilated) Model, CMAM-DAS, which has hourly values, and compares these with analysis using the full model. Fits to $60 \mathrm{~d}$ intervals at the equator show reasonable agreement between full and SABERsampled data analysis for mean, diurnal and semidiurnal tides, with or without inclusion of a linear detrend term. At $50^{\circ} \mathrm{N}$ where there is irregular coverage of local times, there are serious disagreements in all parameters except the background mean, and detrending has a significant effect.
\end{abstract}

Keywords. Meteorology and atmospheric dynamics (Middle atmosphere dynamics; Waves and tides; Instruments and techniques)

\section{Introduction}

Many papers have taken advantage of the slow precession in local time of the TIMED satellite to analyze SABER temperature data for solar generated tides. A variety of analysis methods have been developed over the years of SABER operation: the simpler 60 day fits of Yuan et al. (2010) a

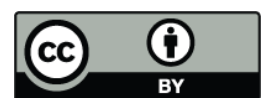

Correspondence to: C. E. Meek

(chris.meek@usask.ca) form of wavelet analysis described by Pancheva et al. (2009), a $120 \mathrm{~d}$ analysis developed by Zhang et al. (2006), and finally a yearly analysis, e.g. Huang et al. (2006, 2010). These methods are mostly based on mathematical arguments and assumptions about the variability of temperature fields and tides. The purpose of this paper is not to test all present methods of SABER tidal analysis but to encourage more testing of such methods with realistic models. The first mention of the use of CMAM-DAS data, as sampled by the SABER instrument, is in Manson et al. (2010). It was briefly noted there, in the context of solar tides as obtained from full CMAM-DAS data, that relatively sparse data as available from SABERlike sampling led to inferior descriptions of the tides at certain heights and months.

The paper is organized as follows. First there is a short section on the sampling process. Next the tidal analysis with optional detrend is described. A short discussion and conclusions follow.

\section{Orbital issues and sampling process}

If the ascending (A) and descending (D) node data are sampled approximately $12 \mathrm{~h}\left(180^{\circ}\right)$ apart in local time (LT), then at the $3^{\circ}$ precession per day, the A node will sample $180^{\circ}$ of local time in $60 \mathrm{~d}$, and the D node will sample the other $180^{\circ}$. This situation most closely applies at the equator. The latitudes $50^{\circ} \mathrm{S}$ to $50^{\circ} \mathrm{N}$ are always sampled, but in order to avoid looking into the sun, the field of view (FOV, perpendicular to the spacecraft orbit, tangent to the earth) is reversed every $60 \mathrm{~d}$, the so-called "yaw maneuver", This changes the LT sample spacing between the A and D nodes, and as will be seen later, even in a non-yaw $60 \mathrm{~d}$ interval at the equator there is still a small LT gap due to the "look geometry".

In order to sample the model atmosphere as SABER would, it is convenient to set up a model orbit which agrees 


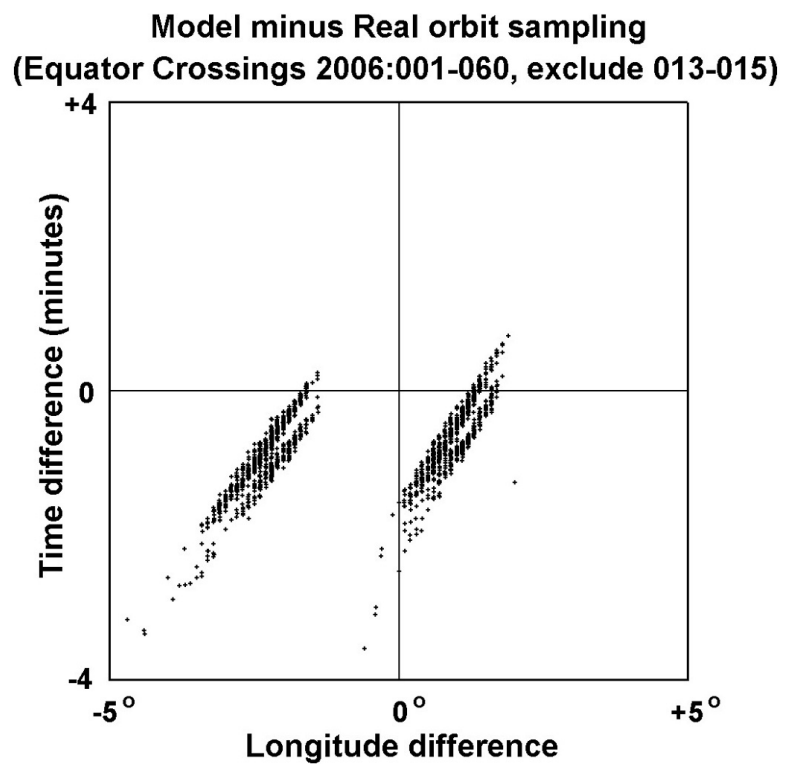

Fig. 1. Model orbit sampling compared with SABER: time and longitude differences over a 60d interval including a yaw maneuver, but excluding days between the (different) model and SABER yaw dates.

closely with the real orbit. This will be an ideal since it can be sampled as close to a wanted latitude as desired and there are no missing values. Such a function has been developed. Figure 1 compares the model and actual SABER sampling over a $60 \mathrm{~d}$ interval, which includes a yaw maneuver, by comparing sample longitude and time differences for equator crossings. Since for 2006 the actual yaw date/time (which varies from year to year) and model yaw date/time differ by about 2 days (e.g. days 13-15 here), equator crossings during this interval are not plotted. The agreement is seen to be within \pm 2 min and $\pm 4^{\circ}$ in longitude, so this sampling scheme is judged to be satisfactory for tidal fits to $60 \mathrm{~d}$ intervals.

Note that the accuracy of tidal fits relies on having data samples more or less evenly distributed in local time. Five cases are illustrated in Fig. 2a-e: equator (no yaw maneuver), equator (with yaw), $50^{\circ} \mathrm{N}$ (looking $\mathrm{N}$ ), $50^{\circ} \mathrm{N}$ (with yaw), and $50^{\circ} \mathrm{N}$ (looking $\mathrm{S}$ ). The model orbit performs yaw maneuvers on days 15 (start looking North), 75(S), 136(N), $258(\mathrm{~S})$, and $320(\mathrm{~N})$. A close to worst case scenario is shown in Fig. 2e. At $50^{\circ} \mathrm{N}$ when the satellite is looking southward the A and D node samples are very close together in LT (at $\sim 52^{\circ} \mathrm{N}$, the maximum northern latitude sampled when the FOV is southward, they would be the same). The $3^{\circ}$ day $^{-1}$ precession only covers $180^{\circ} \mathrm{LT}$ plus the small A/D LT spacing. Another problematic case is during a yaw maneuver for large off-equator latitudes, e.g. Fig. 2d, The samples are very unevenly distributed in LT. Extra weight will be given to some LT intervals, and bias the results.

To return to Fig. 2e, the A and D samples are almost at the same local time, so the advantage of A and D is almost lost, and a seasonal trend can then look like an LT-dependent, or diurnal, variation; that is, tides and trends are coupled. Adding a linear trend to the fit process when there is none in reality, can affect the tidal results. For example if the first LT sample is at a "zero" of a semidiurnal tide, the sampling in LT is of a single oscillation of a sine wave, which itself has a apparent trend, e.g., going from negative to positive over 12:00 LT. On the other hand if the first LT sampled is at a tidal maximum then the value varies, e.g., negative, positive, negative, there is no trend (provided there is only one tidal component present, and samples are evenly distributed in LT). In analysis of real data, trends are not known a priori, so they have to be included as an option just in case. In a $60 \mathrm{~d}$ interval propagating planetary waves can also be aliased into near-tidal frequencies (Meek and Manson, 2009). As mentioned above, a separate problem arises if local times, i.e. tidal phases, are sampled unevenly, or there are large gaps. This is demonstrated later in Sect. 4 and Figs. 4 and 5.

\section{Sampling process with CMAM-DAS}

$6 \mathrm{~h}$ resolution data from CMAM-DAS, e.g. Ren et al. (2008), were made generally available during the recent IPY (International Polar Year), and $1 \mathrm{~h}$ resolution data were later obtained on request. These CMAM-DAS temperature (and wind) data, originally on pressure levels, have been interpolated to geopotential height levels spaced by $3 \mathrm{~km}$. The grid cells are $3.75^{\circ}$ longitude by $\sim 4^{\circ}$ latitude (the latitude spacing varies slightly towards the poles ). "SABER-sampling" is performed by running the model orbit function with a $20 \mathrm{~s}$ step. When the sample location crosses a wanted latitude $\left(-80^{\circ}\right.$ to $80^{\circ}$ step $\left.10^{\circ}\right)$ the exact time and longitude are found by interpolation. Then the temperature value is obtained by tri-linear interpolation of the wanted CMAM-DAS parameter in longitude, latitude, and time within the bracketing grid cell. For full CMAM-DAS analysis, rather than interpolating to specific latitudes, the closest available latitude is used.

\section{Tidal analyses}

For simplicity just diurnal and semidiurnal migrating tides are considered. Two versions of least squares fit were used

$$
\begin{aligned}
T_{i}= & A_{0}+A_{24} \cos \left(\omega_{24} t_{i}+m_{24} \ell_{i}-\phi_{24}\right) \\
& +A_{12} \cos \left(\omega_{12} t_{i}+m_{12} \ell_{i}-\phi_{12}\right)
\end{aligned}
$$

where $t_{i}$ is time in hours, $\omega_{24}=2 \pi / 24, \omega_{12}=4 \pi / 24, \ell_{i}$ is East longitude in radians, and $T_{i}$ is temperature for sample $i$; diurnal and semidiurnal wavenumbers are $m_{24}=1, m_{12}=2$. Unknowns are the background mean $A_{0}$, the diurnal amplitude and phase, $A_{24}, \phi_{24}$, and the semidiurnal amplitude and phase, $A_{12}, \phi_{12}$.

The second version adds a time trend, $B$, to account for seasonal change over the required $60 \mathrm{~d}$ fit intervals. 
Local time coverage over selected 60d intervals for 2 latitudes
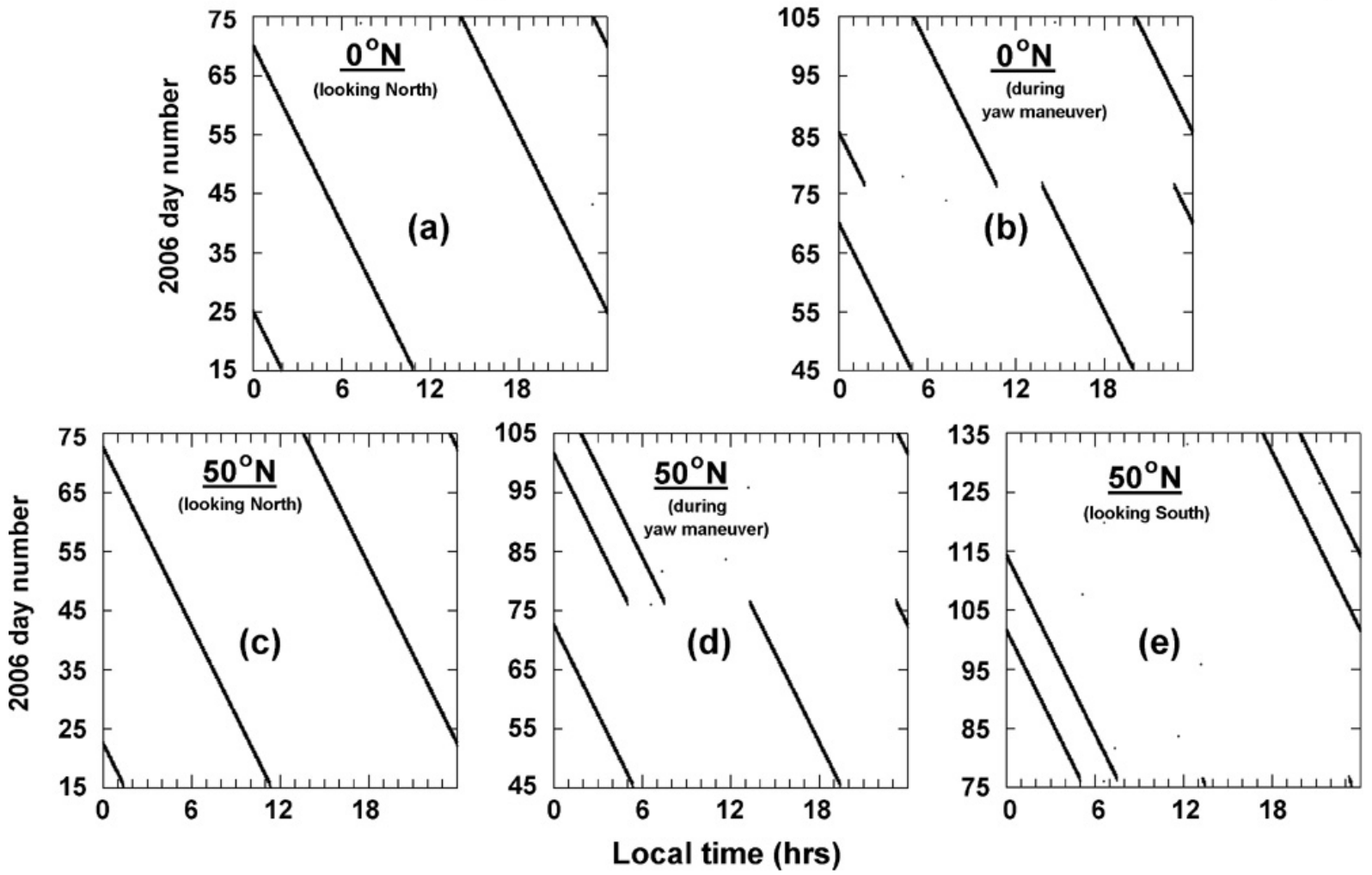

Fig. 2. Local time coverage for $60 \mathrm{~d}$ intervals with and without yaw maneuver at $0^{\circ}$ and $50^{\circ} \mathrm{N}$.

$$
\begin{aligned}
T_{i}= & A_{0}+A_{24} \cos \left(\omega_{24} t_{i}+m_{24} \ell_{i}-\phi_{24}\right) \\
& +A_{12} \cos \left(\omega_{12} t_{i}+m_{12} \ell_{i}-\phi_{12}\right)+B t_{i}
\end{aligned}
$$

Both fits minimize squared error in temperature, $T$.

There were insignificant differences between the full CMAM-DAS no-detrend, and linear-detrend analysis - in fact the only differences seen in the plots were in the $24 \mathrm{~h}$ amplitude for $50^{\circ} \mathrm{N}$, and they were less than one contour level. Consequently we show only the no-detrend analyses for full CMAM-DAS. Likewise the background means from the full CMAM-DAS and SABER-sampled no-detrend and detrend agreed very well, so they are also not shown. In the following contour plots (Figs. 3, 4) note that the $60 \mathrm{~d}$ intervals are (closely) centred on calendar months that is " $\mathrm{J}$ (anuary)" stands for day 351 to 45 (yaw maneuver at day 15), "F(ebruary)" is days 15-75 (no yaw, "looking North"), "M(arch)" days 45-105 (yaw at day 75), "A(pril)" days 75-135 (no yaw "looking South") etc. Therefore the biggest differences between full CMAM-DAS and SABERsampled are expected when the interval sampling is upset by a yaw maneuver (e.g. January, March, May, July, September, November), and also at $50^{\circ} \mathrm{N}$ when SABER looks to the South (e.g. April, August, December).

In the plot, amplitude contour cells are filled by bi-linear interpolation, and for phase this interpolation is done on sin and cos, which are combined to reconstitute the phase. If the corners of a cell have a spread greater than $180^{\circ}$ of period the cell is left as a gap. Note also that some high amplitude areas have been clipped.

\section{Comparison of CMAM-DAS and SABER-sampled tides}

Figure 3 compares equatorial migrating tides between full CMAM-DAS and SABER-sampled no-detrend, and lineardetrend. There is generally good agreement for the diurnal amplitudes and phases; except that SABER-sampled amplitudes artificially distinguish a weaker maximum near $82 \mathrm{~km}$ in the late summer and fall. The SABER-sampled phases are more irregular with time below $\sim 73 \mathrm{~km}$. The SABERsampled semidiurnal tidal amplitudes are more structured and larger, from fall through winter and including spring, especially below $80 \mathrm{~km}$, than the fully sampled CMAMDAS. Semidiurnal phases from the all-times and all- locations CMAM provide regular and smooth (height and time) annual variations, with maxima occurring earlier in the UT day during summer centred months; SABER sampling has much greater monthly variations, consistent with the amplitude structure. With regard to amplitudes above $80 \mathrm{~km}$ at the equator, the October-centred maxima of amplitudes 


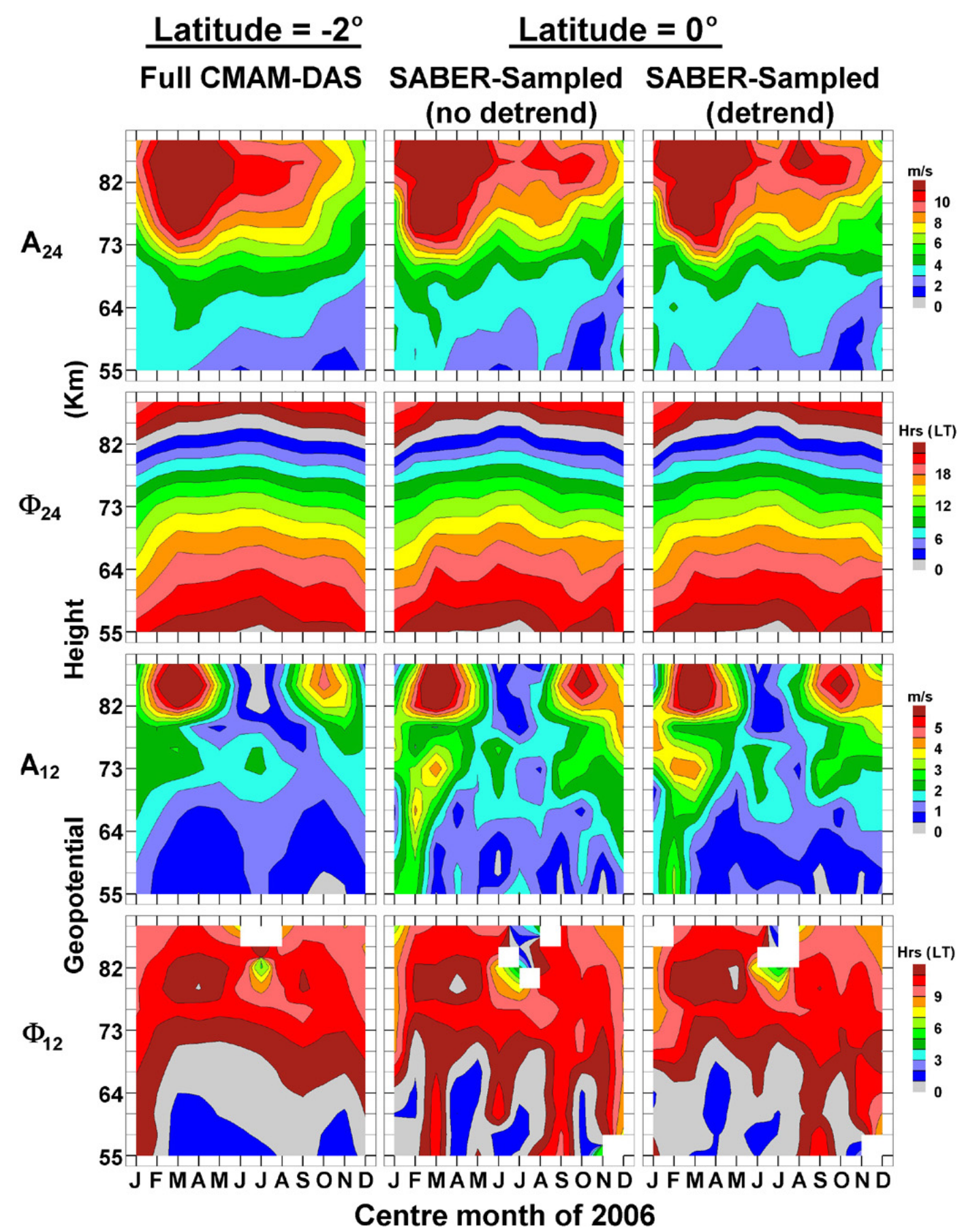

Fig. 3. Tidal analysis of Full CMAM-DAS data (no detrend) with SABER-sampled CMAM-DAS (without and with detrend) at latitudes $-2^{\circ} \mathrm{N}$ and $0^{\circ}$, respectively. Fits of 24 and $12 \mathrm{~h}$ tides to $60 \mathrm{~d}$ intervals centred on calendar months. Mean (and trend in the right hand column) were included in the least squares fit, but just the amplitude and hour of maximum are shown.

are over emphasized in the SABER-sampled analysis. The differences between samplings of semidiurnal amplitudes in February are difficult to explain, as the LT sampling by SABER is close to uniform. However the background mean (not shown) does have a minimum centred on February rather than a smooth trend.
Figure 4 compares the tidal values at $50^{\circ} \mathrm{N}$. There are very significant differences between results from SABER orbit sampling and those from the full CMAM-DAS analysis: there are "hot spots" in the diurnal tide amplitudes, winter phase-height profiles are quite irregular and even show some upward phase propagation. But there are some diurnal phase 


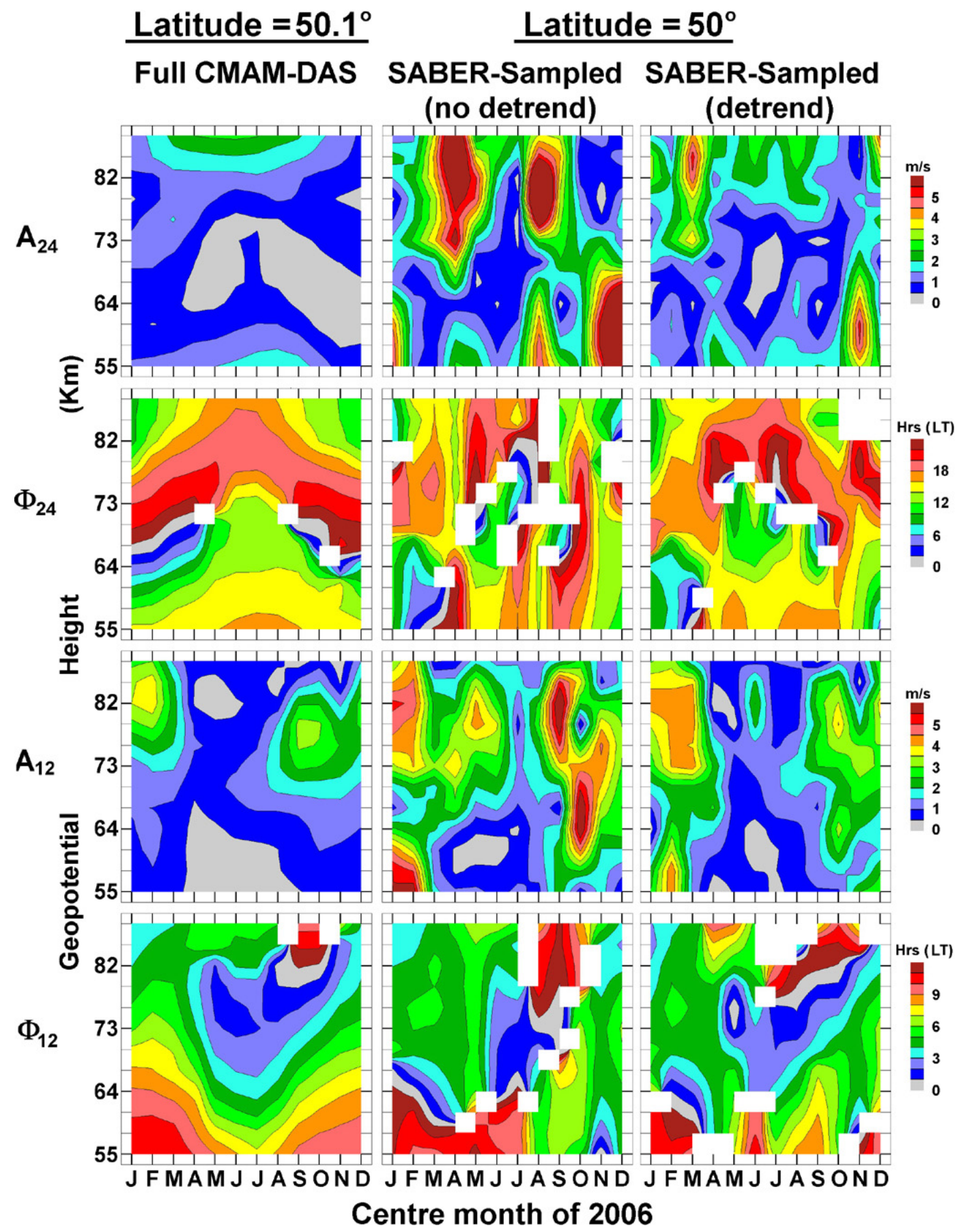

Fig. 4. As in Fig. 3 but at latitudes $50.1^{\circ} \mathrm{N}$ and $50^{\circ} \mathrm{N}$, respectively.

similarities with the full CMAM-DAS at the greatest heights. The semidiurnal amplitude also shows hot spots, but these are somewhat subdued by the detrend analysis. The latter has an overall similarity with the full CMAM-DAS although they differ in many details. The winter phase is quite variable and shows unrealistic upward phase propagation at the beginning of the year, a major change from the full CMAM-DAS analysis.
Figure 5 examines a hot spot in the SABER-sampled diurnal tide seen in Fig. 4, April, in terms of sample times relative to the tidal phase. It can be seen that upper height sampling only covers about half the tidal oscillation, and apart from a few isolated points is centred near a zero crossing. Thus the maxima are poorly defined and the squared error is unable to sufficiently control their magnitudes in the fitting algorithm. This is one possible reason for their large values. 
Full CMAM-DAS Diurnal tidal phase: SABER sampling. (2006: 60d centred on April)

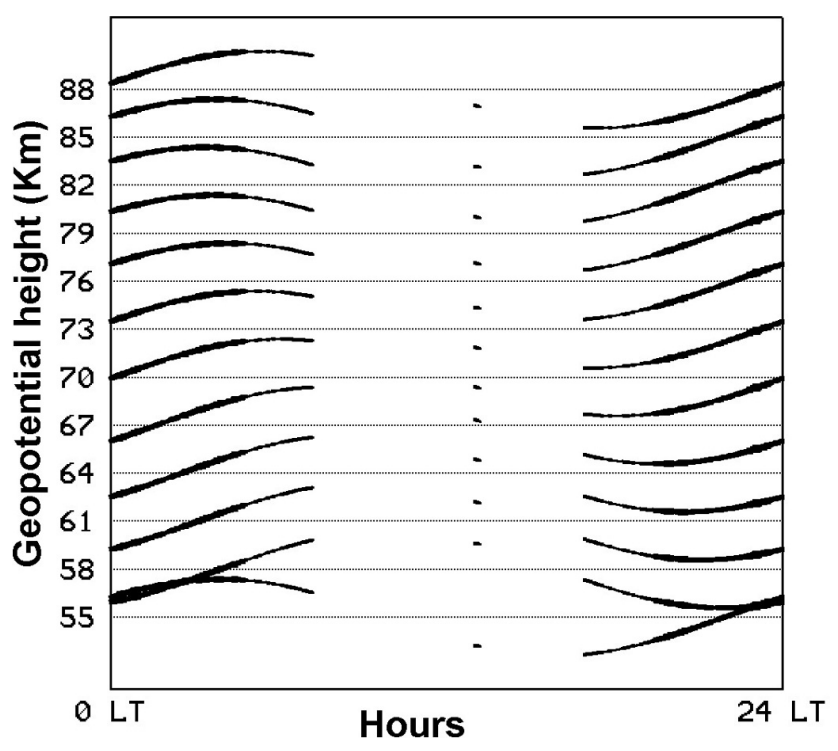

Fig. 5. Sine waves with phase equal to full CMAM-DAS analysis at each height for $50.1^{\circ} \mathrm{N}$ are plotted with a thickness proportional the number of SABER samples in the $60 \mathrm{~d}$ interval to show the weighting inherent in the least squares analysis.

\section{Longitude mean subtraction?}

Although the purpose of this paper is not to test various methods, a particularly interesting variant has been presented by Forbes et al. (2008). Although some details are not clear, and it would be useful to exactly duplicate the method on the CMAM-DAS model, the process seems to be roughly this: e.g. for one latitude SABER data for a $60 \mathrm{~d}$ interval and given latitude band are binned in longitude, and then Fourier analysed to identify the zonal mean and stationary waves, viz. the "constant" value at each longitude. This interval is slid by $1 \mathrm{~d}$, and subtracted from the original ascending (A) and descending (D) nodes of the raw data. with the intention of removing the mean, stationary waves, and time varying seasonal tends, which can alias into tidal frequencies. In the present work, it seems that a similar analysis can be obtained by removing $60 \mathrm{~d}$ longitude means separately, which removes stationary waves, and then including a time trend with the tide fit to reduce aliasing. But propagating PW are not removed by this process because their contribution to a particular longitude bin over $60 \mathrm{~d}$ is not a constant. Also in order for this procedure to not affect the tide, each longitude bin for A and D must separately have all phases of the tide of interest represented approximately equally; otherwise the bin-average will contain some tidal information, which will be subtracted from the data set.

Although the information is contained in Fig. 2, a clearer way to test these requirements for migrating tides is with lo- cal time (LT) plotted against longitude as in Fig. 6. There are four panels each containing 4 sub plots. Each panel represents a different $60 \mathrm{~d}$ time interval (an interval covering a Yaw maneuver and an interval with SABER looking southward) and/or latitude $\left(0\right.$ and $\left.50^{\circ} \mathrm{N}\right)$. The four subplots show sampling versus local time for $\mathrm{A}$ and $\mathrm{D}$ nodes, and diurnal ("24h") and semi-diurnal (" $12 \mathrm{~h}$ ") local times. The local time axes represent full tidal phase cycles, and show how much of the particular cycle is being sampled at each longitude.

For the migrating diurnal tide during $60 \mathrm{~d}$ at the equator, it can be seen that A and D each cover a different half (approximately) of the cycle, so the longitudinal means subtracted will both partially remove tidal information. At $50^{\circ} \mathrm{N}$, because the chosen "no Yaw" interval is for SABER looking southward, the A and D nodes cover approximately the same part of the phase cycle, leaving a large gap. Again tidal information is removed. When this $\mathrm{A}$ and $\mathrm{D}$ longitude mean subtraction method was tested on the data shown in Figs. 3 and 4, the SABER-sampled diurnal tide ampltude and phase exhibited very strong month-to-month oscillatory artifacts at most heights (not shown), while the semi-diurnal was less modified, but not improved. The inclusion or not of linear detrending during the tidal fit made little difference to the agreement with the full model fit.

\section{Conclusions}

The comparison between tides from full and SABERsampled equator data is very good in all parameters except the semidiurnal amplitude, where there is a significant difference near $\sim 73 \mathrm{~km}$ in February-March whether or not the trend is included (phases at these locations are also less than good). This may be due to a varying background trend.

At $50^{\circ} \mathrm{N}$ there are major differences between full and SABER-sampled data. The SABER-sampled data show large amplitude spikes especially in the diurnal but also in the semidiurnal component, and phase discrepancies in both tides that are large enough to change the sign of the vertical wavelength from upward to downward propagation, e.g. January-March in the semidiurnal component. SABERsampled data also provide tidal phases that vary significantly and erratically by month, quite unlike the relatively smooth annual variations of phases from the full sampling of CMAM-DAS. Detrending the SABER data does improve the comparisons somewhat, if not the vertical phase gradients.

Discrepancies such as these are to be expected with analysis of a sparsely sampled geophysical parameter. The only way to identify potential problems is by similar sparse sampling of realistic data from physically rich General Circulation Models, which have sufficiently high time resolution so that accurate model values of all the parameters of interest, particularly tides and planetary waves, can be found in advance. 


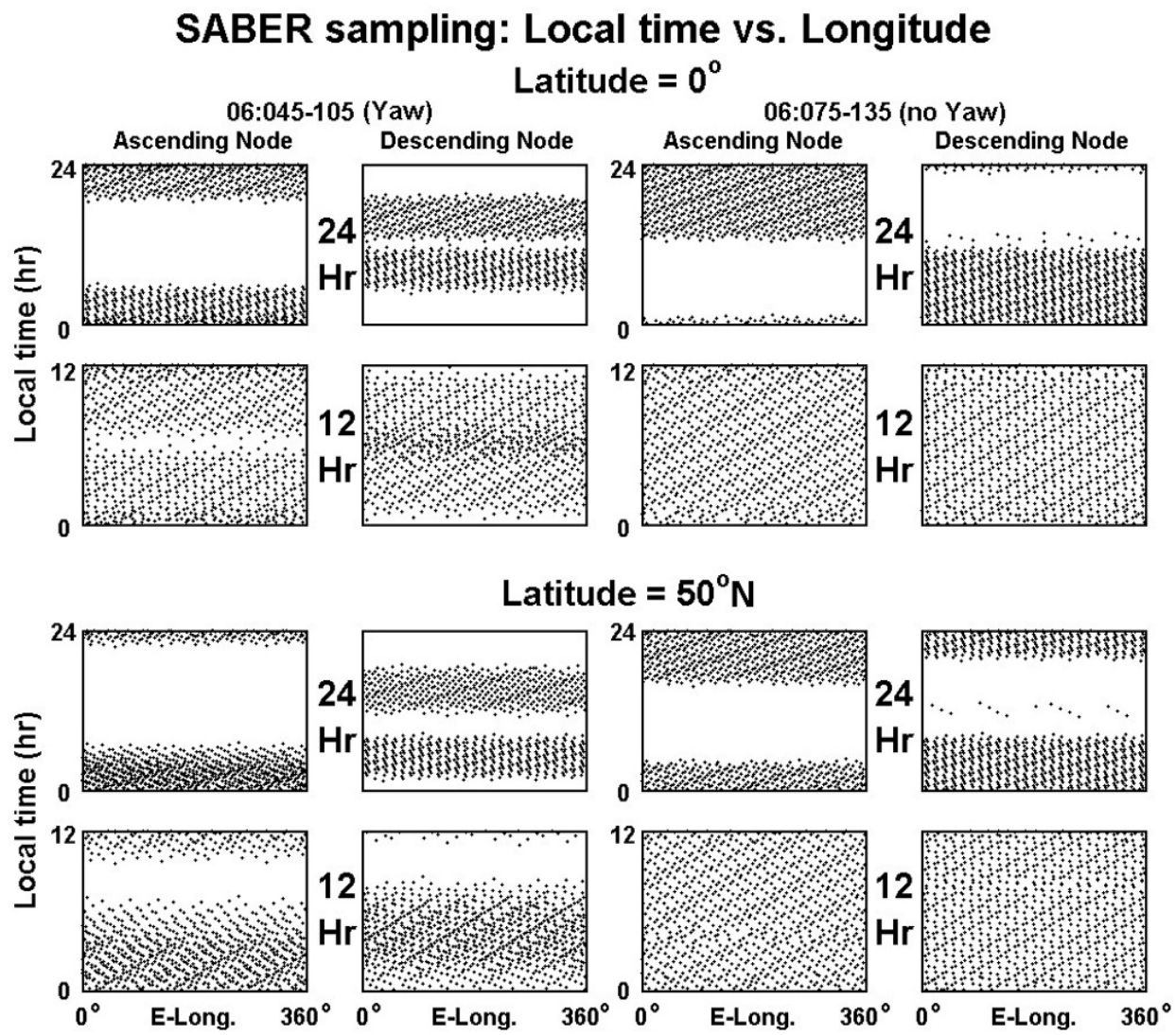

Fig. 6. SABER sampling: Local time vs. Longitude for ascending and decending nodes, migrating diurnal (24h) and semi-diurnal (12 h) tides, without and with Yaw maneuver, 0 and 50 degree Latitudes, to show effect of removing ascending and descending node $60 \mathrm{~d}$ longitude means. The "no YAW" interval shown is for SABER looking southward, i.e. a worst case for $50^{\circ} \mathrm{N}$.

Acknowledgements. Funding for this work was from CANDACPEARL, in part from its IPY funding; the Canadian Natural Sciences and Research Council (NSERC); and the University of Saskatchewan through support to ISAS. We are grateful to the University of Toronto and Environment Canada for access to the $6 \mathrm{~h}$ CMAM-DAS data as part of IPY, and they in turn acknowledge funding sources CFCAS (Canadian Foundation for Climate and Atmospheric Sciences) and the CSA (Canadian Space Agency). We also appreciate the help of Michael Neish (University of Toronto) in providing access to the CMAM-DAS $1 \mathrm{~h}$ data.

Topical Editor P. Drobinski thanks one anonymous referee for her/his help in evaluating this paper.

\section{References}

Forbes, J. M., Zhang, X., Palo, S., Russell, J., Mertens, C. J., and Mlynczak, M.: Troposphere-thermosphere tidal coupling as measured by the SABER instrument on TIMED during July-September 2002, J. Geophys. Res., 113, A02310, doi:10.1029/2005JA011492, 2008.

Huang, F. T., Mayr, H. G., Reber, C. A., Killeen, T., Russell, J., Mlynczak, M., Skinner, W., and Mengel, J.: Diurnal variations of temperature and winds inferred from TIMED and UARS measurements, J. Geophys. Res. 111, A10s04, doi:10.1029/2005JA011426, 2006.

Huang, F. T., McPeters, R., Bhartia, P., Mayr, H., Frith, S., Russell III, J. M., and Mlynczak, M.: Temperature diurnal variations (migrating tides) in the stratosphere and lower mesosphere based on measurements from SABER on TIMED, J. Geophys. Res., 115, D16121, doi:10.1029/2009JD013698, 2010.

Manson, A., Meek, C., and Xu, X.: Comment on "Global structure, seasonal and interannual variability of the migrating semidiurnal tide seen in the SABER/TIMED temperatures (20022007)" by Pancheva et al. (2009), Ann. Geophys., 28, 665-676, doi:10.5194/angeo-28-665-2010, 2010.

Meek, C. E. and Manson, A. H.: Summer planetary-scale oscillations: aura MLS temperature compared with ground-based radar wind, Ann. Geophys., 27, 1763-1774, doi:10.5194/angeo-271763-2009, 2009.

Pancheva, D., Mukhtarov, P., and Andonov, B.: Global structure, seasonal and interannual variability of the migrating semidiurnal tide seen in the SABER/TIMED temperatures (2002-2007), Ann. Geophys., 27, 687-703, doi:10.5194/angeo-27-687-2009, 2009.

Ren, S., Polavarapu, S. M., and Shepherd, T. G.: Vertical propagation information in a middle atmosphere data assimilation system by gravity-wave drag feedbacks, Geophys. Res. Lett., 35, L06804, doi:10.1029/2007GL032699, 2008. 
Yuan, T., She, C., Krueger, D., Reising, S., Zhang, X., and Forbes, J. M.: A collaborative study on temperature diurnal tide in the midlatitude mesopause reagion $\left(41^{\circ} \mathrm{N}, 105^{\circ} \mathrm{W}\right)$ with $\mathrm{Na}$ lidar and TIMED/SABER observations., J. Atmos. Solar-Terr. Phys., 72, 541-549, 2010.
Zhang, X., Forbes, J. M., Hagan, M., Russell III, J. M., Palo, S., Mertens, C., and Mlynczak, G.: Monthly tidal temperatures 20$120 \mathrm{Km}$ from TIMED/SABER, J. Geophys. Res., 111, A10S08, doi:10.1029/2005JA011504, 2006. 